\title{
The genome sequence of the capnophilic rumen bacterium Mannheimia succiniciproducens
}

\author{
Soon Ho Hong ${ }^{1,6}$, Jin Sik Kim ${ }^{1,6}$, Sang Yup Lee ${ }^{1}$, Yong Ho In ${ }^{2,5}$, Sun Shim Choi ${ }^{2}$, Jeong-Keun Rih $^{3}$, \\ Chang Hoon $\mathrm{Kim}^{3}$, Haeyoung Jeong ${ }^{3,4}$, Cheol Goo Hur ${ }^{3}$ \& Jae Jong Kim ${ }^{4}$
}

The rumen represents the first section of a ruminant animal's stomach, where feed is collected and mixed with microorganisms for initial digestion. The major gas produced in the rumen is $\mathrm{CO}_{2}(65.5 \mathrm{~mol} \%)$, yet the metabolic characteristics of capnophilic $\left(\mathrm{CO}_{2}\right.$-loving) microorganisms are not well understood. Here we report the 2,314,078 base pair genome sequence of Mannheimia succiniciproducens MBEL55E, a recently isolated capnophilic Gram-negative bacterium from bovine rumen, and analyze its genome contents and metabolic characteristics. The metabolism of $\boldsymbol{M}$. succiniciproducens was found to be well adapted to the oxygen-free rumen by using fumarate as a major electron acceptor. Genome-scale metabolic flux analysis indicated that $\mathrm{CO}_{2}$ is important for the carboxylation of phosphoenolpyruvate to oxaloacetate, which is converted to succinic acid by the reductive tricarboxylic acid cycle and menaquinone systems. This characteristic metabolism allows highly efficient production of succinic $\stackrel{2}{J}$ acid, an important four-carbon industrial chemical.

The rumen is a fermentation vat in which the feed is collected immediately after being swallowed and is digested through the action of microorganisms. The different microorganisms present in the bovine rumen are able to digest various substrates such as cellulose, hemicellulose, starch, sugar, protein and lipid. Cellulose and hemicellulose are converted to glucose, which is further converted by microbial fermentation to volatile fatty acids (VFAs) such as acetic, propionic and butyric acids ${ }^{1}$. The VFAs generated in the rumen are absorbed through the rumen wall, and used for milk production and energy generation.

We have isolated a novel capnophilic bacterium, Mannheimia succiniciproducens MBEL55E, from bovine rumen ${ }^{2}$. M. succiniciproducens is a non-spore-forming, mesophilic, gram-negative coccobacillus that produces VFAs under the anaerobic conditions present in the rumen. Most notably, it efficiently fixes $\mathrm{CO}_{2}$ and produces substantial amounts of succinic acid ${ }^{2}$.

Various carbohydrates produced during digestion of the feed (e.g., glucose from cellulose by cellulose-digesting microorganisms) are converted into succinic acid as well as acetic, formic and lactic acids by $M$. succiniciproducens ${ }^{2}$. Acetic acid is a major VFA constituting $50 \%-60 \%$ of the total ${ }^{1}$. A major gas present in the rumen is $\mathrm{CO}_{2}$ ( $65.5 \mathrm{~mol} \%$ ), which is a by-product of microbial digestion of nutrients. Therefore, capnophilic bacteria may be important in regulating the overall fermentation profiles within the rumen. Succinic acid is a dicarboxylic acid produced as an intermediate of the tricarboxylic acid (TCA) cycle and also as one of the fermentation products of anaerobic metabolism. It can be used as an ion chelator, food additive, supplement to pharmaceuticals, and as a green feedstock for the manufacture of environmentally friendly polymers, chemical intermediates and solvents ${ }^{3}$. Because succinic acid is produced by carboxylation of three-carbon $\left(\mathrm{C}_{3}\right)$-compounds, capnophilic bacteria contribute most heavily to the production of succinic acid in the rumen. Succinic acid can be further converted to propionic acid, which constitutes about $20 \%$ of total VFAs, by other ruminal microorganisms ${ }^{1}$. Even though the metabolic characteristics of cellulose-degrading microorganisms, acetic acid producers and methanogens have been extensively studied, those of rumen capnophilic bacteria have received little attention.

We have sequenced and analyzed the genome of a rumen capnophilic bacterium M. succiniciproducens MBEL55E. To understand the metabolic characteristics of $M$. succiniciproducens, we carried out genome-scale metabolic flux analyses (MFA) using the in silico metabolic pathways constructed directly from the genome sequence and actual fermentation profiles. Also, comparative genome analyses were carried out with several related bacteria including Pasteurella multocida Pm70, Haemophilus influenzae Rd, Escherichia coli K-12 and Bacillus subtilis. Based on these analyses, we suggest that M. succiniciproducens is a highly efficient succinic acid producer.

\section{RESULTS}

General genome features

The M. succiniciproducens genome is a single circular chromosome of 2,314,078 base pairs (bp) with no plasmid (Fig. 1). General features

\footnotetext{
${ }^{1}$ Metabolic and Biomolecular Engineering National Research Laboratory, Department of Chemical and Biomolecular Engineering, Department of BioSystems, BioProcess Engineering Research Center and Bioinformatics Research Center, Korea Advanced Institute of Science and Technology, 373-1 Guseong-dong, Yuseong-gu, Daejeon 305-701, Republic of Korea. 'Bioinfomatix, Inc., The fifth floor, Nam Chang Bldg., 748-162 Yeoksam-dong, Gangnam-gu, Seoul 135-925, Republic of Korea. ${ }^{3}$ Korea Research Institute of Bioscience and Biotechnology (KRIBB), 52 Oun-dong, Yuseong-gu, Daejeon 305-333, Republic of Korea. ${ }^{4}$ GenoTech Corp., 461-6 Jeonmin-dong, Yuseong-gu, Daejeon 305-390, Republic of Korea. ${ }^{5}$ IDRTech Inc., 461-6 Jeonmin-dong, Yuseong-gu, Daejeon 305-390, Republic of Korea.

${ }^{6}$ These authors contributed equally to this work. Correspondence should be addressed to S.Y.L. (leesy@kaist.ac.kr).
}

Published online 19 September 2004; doi:10.1038/nbt1010 
Figure 1 Circular representation of the M. succiniciproducens MBEL55E genome. The outermost circle indicates the chromosomal location in bp (each tick is $100 \mathrm{~kb}$ ). The distribution of genes is represented by colored boxes according to the functional category in the COG database and direction of transcription (outer band indicates forward direction; inner band indicates reverse direction). Red and green arrows indicate the 강 locations and direction of rRNA and tRNA genes, respectively. The colored inner circle represents the GC skew value (blue indicates positive value and red indicates negative value). The innermost circle shows the $\mathrm{G}+\mathrm{C}$ content (higher values outward).

of the genome are summarized in Table 1. The average $\mathrm{G}+\mathrm{C}$ content is $42.5 \%$. The first base of the gidA gene was assumed to be the origin of replication based on the lower bound of the cumulative GC skew and the inversion of the GC skew value ${ }^{4}$ (see Supplementary Fig. 1 online). Gene prediction and annotation identified 2,384 open reading 윽 frames (ORFs) with an average length of $873 \mathrm{bp}$; we assigned putative functions to $1,471(61.7 \%)$ of the ORFs. Of the remaining 을 913 ORFs, 360 (15.1\%) showed similarity to ORFs encoding hypothetical proteins of unknown function annotated in other genomes, whereas $553(23.2 \%)$ had no significant similarity (using the cutoff E-value of $10^{-5}$ ) to data in the public databases (see Supplementary Table 1 online). The total length of the protein coding region is $2,079,969 \mathrm{bp}$, which covers $89.9 \%$ of the chromosome (see Supplementary Fig. 1 online). There were 19 rRNAs, including six 16S, six N $23 \mathrm{~S}$ and seven $5 \mathrm{~S}$ rRNAs, as well as 60 tRNAs covering all 20 amino acids. No pseudogenes or spliced genes were found.

\section{Comparative genome analysis}

M. succiniciproducens is a nonpathogenic capnophilic rumen bacterium, which, on the basis of $16 \mathrm{~S}$ rRNA sequence alignment, is considered closely related to the pathogenic strain $P$. multocida Pm70 (ref. 2). Each of the 2,384 ORFs of M. succiniciproducens was compared with the annotated genes in the public databases using BLASTP (Fig. 2). More than one-third (38.6\%, 920 ORFs) of the ORFs showed the greatest similarity to the genes of $P$. multocida, and $21.7 \%$ (517 ORFs) to those of $H$. influenzae (Fig. 2). From these results, M. succiniciproducens was categorized into group $\mathrm{H}$ (Pasteurellaceae) of the clusters of orthologous groups (COG) database. The ORFs of M. succiniciproducens were functionally classified based on the COG database, and were compared with the ORFs of $P$. multocida, E. coli and B. subtilis in more detail (see Supplementary Table 2 online). The M. succiniciproducens genome had fewer ORFs than E. coli $(4,288 \mathrm{ORFs})$ and B. subtilis (4,100 ORFs), but more than P. multocida $(2,014 \mathrm{ORFs})^{5-7}$. The distribution of ORFs based on functional categories was similar to that of the most closely related strain, $P$. multocida. The numbers of ORFs involved in cell envelope biosynthesis (code $\mathrm{M}$ ), cell motility (code $\mathrm{N}$ ), signal transduction (code $\mathrm{T}$ ), energy production and conversion (code $\mathrm{C}$ ), carbohydrate transport and metabolism (code G), amino acid transport and metabolism (code E), and biosynthesis and catabolism (code Q) were similar to those in P. multocida, but were much lower than those in E. coli or B. subtilis. M. succiniciproducens and P. multocida were found to possess relatively higher percentages of genes involved in translation, ribosomal structure and biogenesis (code J). The percentage and number of genes involved in signal transduction mechanisms were considerably lower than those in E. coli and B. subtilis.

Analysis of regional $\mathrm{G}+\mathrm{C}$ content variation from the average $\mathrm{G}+\mathrm{C}$ content of the genome revealed that the M. succiniciproducens genome contains one distinct region or genomic island. This genomic island of about $40 \mathrm{~kb}$ having a $\mathrm{G}+\mathrm{C}$ content of $30 \%$ shows as much as $12.5 \%$ of $\mathrm{G}+\mathrm{C}$ content deviation from the core genome. However, the 44 ORFs found in this island did not show significant homologies (using the cutoff E-value of $10^{-5}$ ) with the annotated genes of any

Table 1 General features of M. succiniciproducens MBEL55E genome

Size (base pairs, bp) $2,314,078$

$\mathrm{G}+\mathrm{C}$ content $(\%)$

Protein-coding sequences (CDS)

No. similar to known genes

1,471

No. similar to genes of general function

No. similar to genes of unknown function

No. of genes not in COG database

553

Total CDSs

2,384

Coding density (\%)

89.9

Average CDS size (bp)

873

No. of $16 \mathrm{~S}-23 \mathrm{~S}$ rRNA

No. of $5 \mathrm{~S}$ rRNA

No. of tRNA 
Figure 2 Comparison of $M$. succiniciproducens MBEL55E ORFs with other organisms. Each of the 2,384 predicted ORFs of $M$. succiniciproducens was used as a query sequence against the COG database. The inset shows the results of a dot plot comparison between the genome sequences of M. succiniciproducens and P. multocida. Abbreviations are: Rpr, Rickettsia prowazekii Madrid E; Pho, Pyrococcus horikoshii OT3; ते Pab, Pyrococcus abyssi; Mpn, Mycoplasma pneumoniae M129; jHp, Helicobacter pylori J99; $\mathrm{Hal}(\mathrm{Hbs})$, Halobacterium sp. NRC-1; Buc, Buchnera sp. APS; Mtu, Mycobacterium tuberculosis H37Rv (lab strain); Mja, Methanococcus jannaschii DSM2661; Hpy, Helicobacter pylori 26695; Mth, Methanobacterium thermoautotrophicum deltaH; Aae, Aquifex aeolicus VF5; Dra, Deinococcus radiodurans R1; Cje, Campylobacter jejuni NCTC11168; Syn, Synechocystis sp. PCC6803; Spy, Streptococcus pyogenes M1; Xfa, Xylella fastidiosa 9a5c; Vch, Vibrio cholerae El Tor N16961 (serotype 01); Lla, Lactococcus lactis IL1403; Tma, Thermotoga maritima MSB8; Ccr, Caulobacter crescentus; Mlo, Mesorhizobium loti MAFF303099; Nma, Neisseria meningitidis Z2491 (serogroup A); Nme, Neisseria meningitidis MC58 (serogroup B); Bsu, Bacillus subtilis 168; Pae, Pseudomonas aeruginosa PA01; Bha, Bacillus halodurans C-125; Eco, Escherichia coli K-12 MG1655; EcZ(Ece), Escherichia coli 0157:H7 EDL933; Hin, Haemophilus influenzae Rd; Man-S, no homology or M. succiniciproducens-specific; Pmu, Pasteurella multocida PM70.

은

microorganism in the databases and thus there is no evidence of horizontal gene transfer.

M. succiniciproducens and P. multocida, which had the highest number of orthologous genes, were compared for their overall genome structure conservation. Even though only $2.60 \%$ of the M. succiniciproducens genome sequence perfectly matched the $P$. multocida genome sequence, there were weak linear relationships between the two genomes along the two crossed diagonal directions (Fig. 2 and Supplementary Fig. 1 online). Therefore, it can be suggested that these two species are closely related and originated from a common ancestor, but went through different evolutionary processes. Similar (2) dot plot analysis was also carried out with $H$. influenzae, another member of the Pasteurellaceae family, which showed substantial homologies to M. succiniciproducens (see Supplementary Fig. 1 online). The two species matched in $3.55 \%$ of their genome sequences, which is greater than the similarity of M. succiniciproducens with P. multocida, but no apparent conservation of linearity was found.

Gene duplication followed by mutation is an important mechanism for generating new genes and new biological functions to adapt to a particular environment. Genome analysis revealed that 255 genes in M. succiniciproducens were duplicated (see Supplementary Table 2 online). Among them, 167 genes had one duplicate, 38 genes had two duplicates and 49 genes had more than two duplicates. The high copynumber genes were found to encode global transcriptional regulators or permeases of the major facilitator superfamily (ProP). For example, the $l y s R$, a transcriptional regulator gene for lysine metabolism, had the most duplicates. The proP gene had 11 duplicates, which were distributed over the entire genome. Many of the genes involved in transcription, amino acid transport and metabolism, and carbohydrate transport and metabolism were duplicated.

\section{Metabolism}

The genes involved in metabolic pathways were analyzed in detail using the information present in $\mathrm{KEGG}^{8}$ and $\mathrm{EcoCyc}^{9}$ (see Supplementary Table 3 online). Based on these results, a metabolic reaction map was constructed (see Supplementary Fig. 2 online). Most of the central metabolic pathway reactions were present, including almost complete Embden-Meyerhof-Parnas and pentose-phosphate pathways. The TCA cycle was also present, but the succinate dehydrogenase gene was not found. This indicates that $M$. succiniciproducens operates an incomplete or branched TCA cycle. It is notable that the EntnerDoudoroff pathway, which is not present in P. multocida ${ }^{7}$ or $H$. influenzae ${ }^{10}$, was also detected in $M$. succiniciproducens. A number of reactions for anaerobic pyruvate dissimilation, organic acid production and anaplerotic reactions were identified. The glyoxylate shunt was absent. At least three enzymes that interconvert $\mathrm{C}_{3}$ compounds and $\mathrm{C}_{4}$ compounds were found; phosphoenolpyruvate (PEP) carboxykinase and PEP carboxylase interconvert PEP and oxaloacetate, whereas the $\mathrm{NADP}^{+}$-dependent malic enzyme interconverts pyruvate and malate. It is notable that the $\mathrm{NADP}^{+}$-dependent malic enzyme (maeB) is present in $M$. succiniciproducens instead of the $\mathrm{NAD}^{+}$-dependent malic enzyme $(s f c A)$ more frequently found in other organisms.

Most of the pathways for building-block biosynthesis were present, including those for amino acids. In the case of the nucleotide biosynthesis pathway, the initial two steps for synthesizing pyrimidines from aspartate and glutamine were missing. The genes involved in these steps are aspartate carbamoyltransferase ( $p y r B I$ ) and dihydroorotase (pyrC), which are also missing in P. multocida ${ }^{7}$ and $H$. influen$z a e^{10}$. Orotate present in the growth medium can complement pyrimidine auxotrophy. The biosynthetic pathways for some cofactors and vitamins such as coenzyme A, NAD, biotin and thiamin were found to contain too many missing links, and therefore, may not function in M. succiniciproducens. Virulence-related genes such as the leukotoxin and capsule biosynthesis genes were not found.

M. succiniciproducens possesses several $\mathrm{ABC}$ transporters for the transport of carbohydrates, amino acids, long chain fatty acids, dipeptides/oligopeptides, $\mathrm{Fe}^{3+} /$ cobalamin, nickel, manganese/zinc, thiamin, sulfate/molybdate and spermidine/putrescine (see Supplementary Table 1 online). M. succiniciproducens also possesses the Sec machinery for the secretion of protein.

A number of genes involved in the transport of external carbon sources such as $m t l A, f r u A, x y l E$ and malX were found in the genome. 


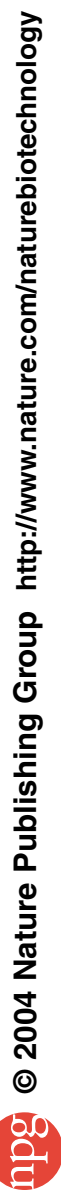

a

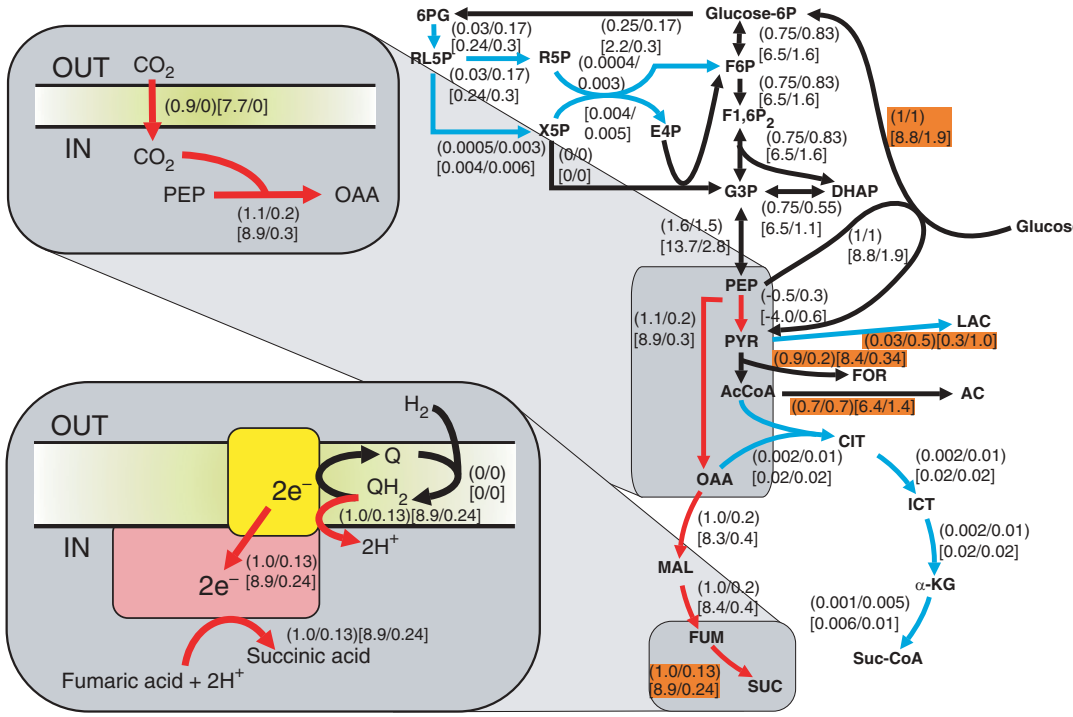

b
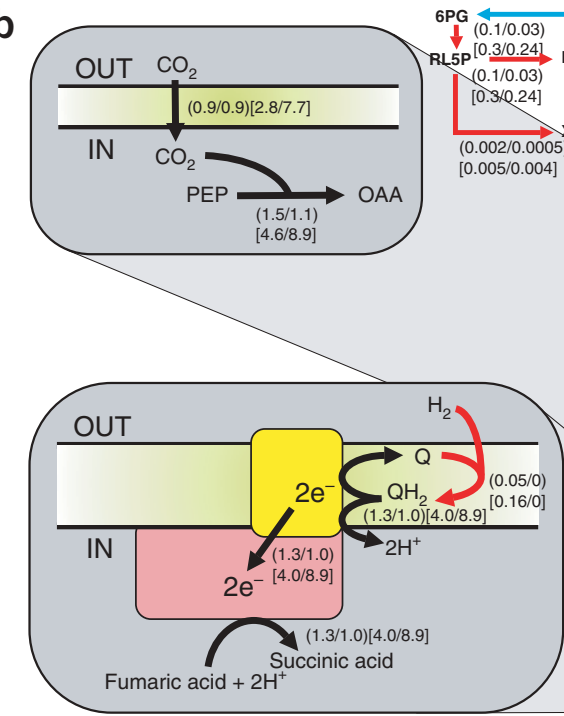

Figure 3 Results of metabolic flux analyses. $(\mathbf{a}, \mathbf{b})$ Graphical representation of normalized intracellular metabolic fluxes ( $\mathrm{mM} / \mathrm{mM}$ glucose) and fluxes not normalized [mM/gDCW/h] for $\mathrm{CO}_{2}$ atmosphere/ $\mathrm{N}_{2}$ atmosphere (a) and $\mathrm{CO}_{2}-\mathrm{H}_{2}$ atmosphere/ $\mathrm{CO}_{2}$ atmosphere (b) during the cultivation of $M$. succiniciproducens in $\mathrm{MH} 2$ medium containing $20 \mathrm{~g} / \mathrm{l}$ glucose at $39^{\circ} \mathrm{C}$ and $\mathrm{pH}$ 5.5-7.5. Red arrows indicate the fluxes that increased by more than twofold, whereas blue arrows indicate the fluxes that decreased to less than half. Orange rectangles indicate fluxes that were measured directly during the fermentations. (c) Profiles of representative intracellular fluxes of $M$. succiniciproducens MBEL55E obtained under $\mathrm{N}_{2}, \mathrm{CO}_{2}$ and $\mathrm{CO}_{2}-\mathrm{H}_{2}$ atmospheres at $\mathrm{pH}$ 5.5-7.5. The glucose consumption rate was not normalized ( $\mathrm{mM} / \mathrm{gDCW} / \mathrm{h}$ ), whereas other fluxes were normalized to the glucose consumption rate ( $\mathrm{mM} / \mathrm{mM}$ glucose) obtained under each condition. 6PG, 6-phosphoglucono-lactone; AC, acetic acid; AcCoA, acetyl-CoA; CIT, citrate; DHAP, dihydroxyacetone-phosphate; E4P, erythrose-4phosphate; $F 1,6 \mathrm{P}_{2}$, fructose-1,6-diphosphate; F6P, fructose-6-phosphate; FOR, formic acid; FUM, fumaric acid; G3P, glyceraldehyde-3phosphate; ICT, isocitrate; $\alpha$-KG, $\alpha$-ketoglutarate; $L A C$, lactic acid; MAL, malic acid; OAA, oxaloacetate; PYR, pyruvate; $Q$, menaquinone; $\mathrm{QH}_{2}$, menaquinol; $\mathrm{R} 5 \mathrm{P}$, ribose-5-phosphate; RL5P, ribulose-5-phosphate; SUC, succinic acid; Suc-CoA, succinyl-CoA; X5P, xylulose-5phosphate; DCW, dry cell weight.
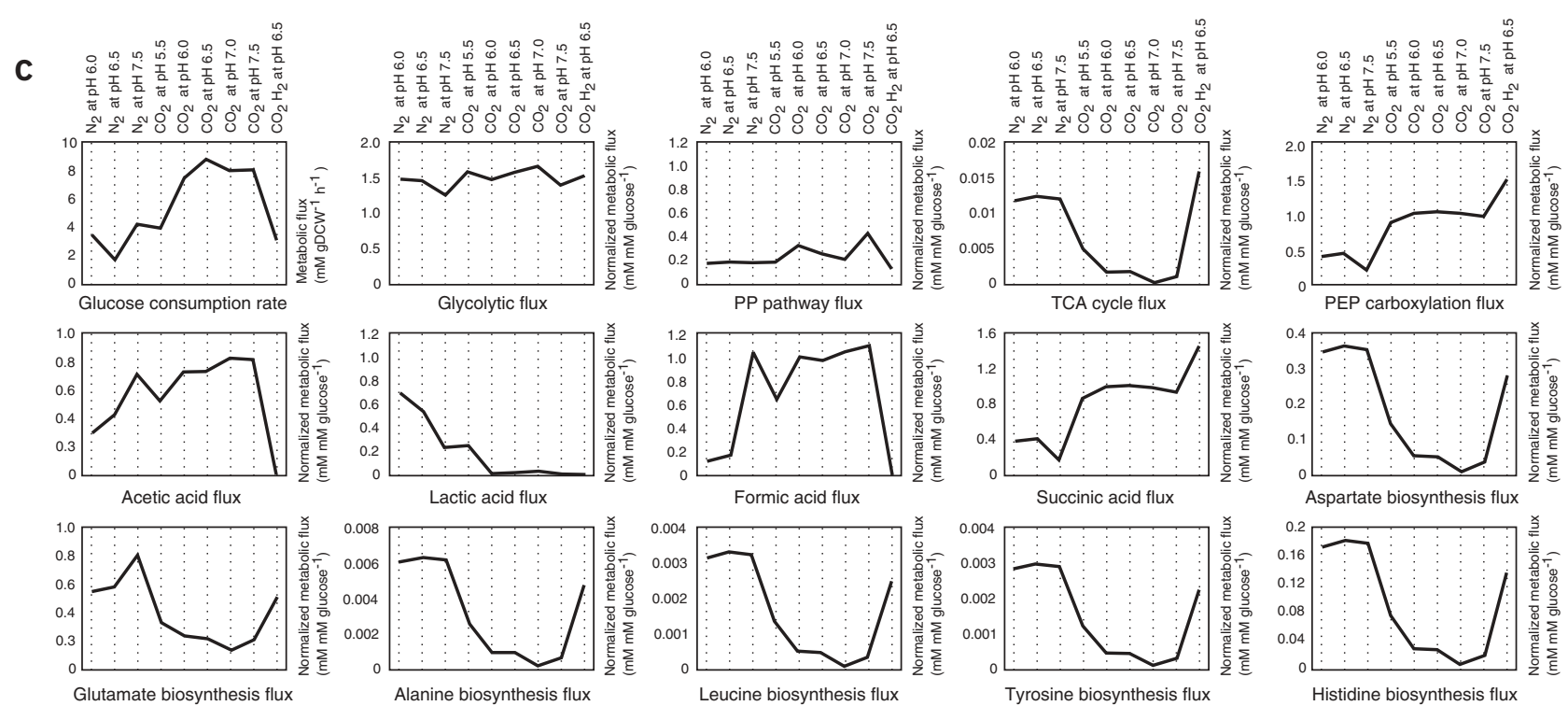
To examine the range of carbon substrates M. succiniciproducens uses, we cultivated the bacterium on various carbon sources. As predicted from the genome sequence, $M$. succiniciproducens used glucose, fructose, xylose, sucrose, maltose, lactose and mannitol (see Supplementary Table 4 online). However, carbohydrate polymers such as cellulose and hemicellulose were not used by M. succiniciproducens, as predicted by the absence of the necessary enzymes. The mannitolpositive growth characteristics support the classification of this strain as a Mannheimia sp. in the Pasteurellaceae family, which also contains Acinobacillus, Pasteurella, Haemophilus and Lonepinella ${ }^{10-13}$.

$M$. succiniciproducens was found to possess a pathway for the synthesis of menaquinone (vitamin $\mathrm{K}_{2}$ ), but not for ubiquinone (coenzyme Q). Ubiquinone is generally used for aerobic respiration, whereas menaquinone is used for anaerobic respiration with electron acceptors other than oxygen and nitrate, such as fumarate. The presence of a menaquinone system, operation of a branched TCA cycle and production of succinic acid even under aerobic conditions all suggest that $M$. succiniciproducens uses fumarate as an electron acceptor and possesses oxygen-independent metabolic characteristics.

To evaluate these hypotheses, we analyzed the respiratory system of M. succiniciproducens and compared it with those of other microorganisms. Respiratory networks of various microorganisms were constructed based on the COG database and were hierarchically clustered for comparison (see Supplementary Fig. 3 online). The 을 respiratory system of $M$. succiniciproducens was most similar to those of P. multocida, H. influenzae, Vibrio cholerae, E. coli and Campylobacter jejuni. M. succiniciproducens lacks genes for nitrate (narI and nar $Y$ ) and oxygen (cyoA) respiration; it also lacks the genes for aerobic fumarase (fumA) and succinate dehydrogenase (sdhA) of the TCA cycle, which convert succinic acid to malic acid under aerobic conditions. The NADH dehydrogenase genes (nuoB, nuoK and $n d h)$ were present in M. succiniciproducens, but not in P. multocida (see Supplementary Fig. 3 online). These results suggest that M. succiniciproducens evolved the respiratory system to adapt to the $\mathrm{CO}_{2}$-rich and $\mathrm{O}_{2}$-deficient rumen condition. As a capnophilic bacterium, N. succiniciproducens efficiently carboxylates PEP to form oxaloace(2) tate, which is converted to fumarate via malate. Fumarate acts as a major electron acceptor as mentioned above, and is converted to succinic acid. This is strongly supported by the finding that M. succiniciproducens possesses anaerobic fumarase, fumarate reductase and $\mathrm{NADH}$ dehydrogenase, which form an efficient respiration network, whereas it lacks the key oxygen respiratory enzymes, succinate dehydrogenase and cytochrome oxidase bo3.

\section{MFA}

To determine the overall genome-scale metabolic characteristics of M. succiniciproducens, we carried out MFA using the in silico metabolic network directly constructed from the annotated genome. The in silico metabolic network of M. succiniciproducens consisted of 373 reactions (121 reversible and 252 irreversible) and 352 metabolites (see Supplementary Table 3 online). The rumen condition and content are dependent on rumen ecology and fermentation balance, both of which are affected by feeding. The gas composition is also an important factor that can influence the microbial population and activities in the rumen ${ }^{1}$. The average composition of rumen gas is: $\mathrm{CO}_{2}, 65.5 \mathrm{~mol} \% ; \mathrm{CH}_{4}, 26.8 \mathrm{~mol} \% ; \mathrm{N}_{2}, 7 \mathrm{~mol} \% ; \mathrm{O}_{2}, 0.5 \mathrm{~mol} \%$; and $\mathrm{H}_{2}, 0.2 \mathrm{~mol} \%$. Therefore, we examined the effects of changes in gas composition on the metabolism of $M$. succiniciproducens by using MFA constrained with actual data derived from cultivating cells under $\mathrm{N}_{2}, \mathrm{CO}_{2}$ and $\mathrm{CO}_{2}-\mathrm{H}_{2}$ atmospheres. We did not examine the effects of $\mathrm{O}_{2}$ and $\mathrm{CH}_{4}$ because $M$. succiniciproducens does not use them.
Similarly, the effects of $\mathrm{pH}$ were examined by cultivating cells at $\mathrm{pH}$ values of 5.5 to 7.5. We obtained the metabolic flux distributions under various conditions by linear optimization using these fermentation data as constraints (see Supplementary Fig. 4 online), and examined them by Bayesian network analysis.

As predicted above, M. succiniciproducens was found to operate a branched TCA cycle (Fig. 3a), which is usually observed in bacteria that favor anaerobic conditions. The effect of $\mathrm{CO}_{2}$ on the metabolism of $M$. succiniciproducens was examined by comparing the MFA results obtained under $\mathrm{CO}_{2}$ versus $\mathrm{N}_{2}$ growth conditions (Fig. 3a). Under $\mathrm{N}_{2}$ atmosphere, cell growth and glucose consumption rates were much lower, suggesting that $M$. succiniciproducens, like other capnophilic bacteria, relies on a $\mathrm{CO}_{2}$-dependent metabolism. The glycolytic flux under $\mathrm{CO}_{2}$ was four times greater than that obtained under $\mathrm{N}_{2}$. It is notable that the fluxes of PEP carboxylation and the reductive branch of the TCA cycle considerably increased under $\mathrm{CO}_{2} \cdot \mathrm{NAD}^{+}$was regenerated mostly through the reductive branch of the TCA cycle under $\mathrm{CO}_{2}$ atmosphere, suggesting that $M$. succiniciproducens uses a
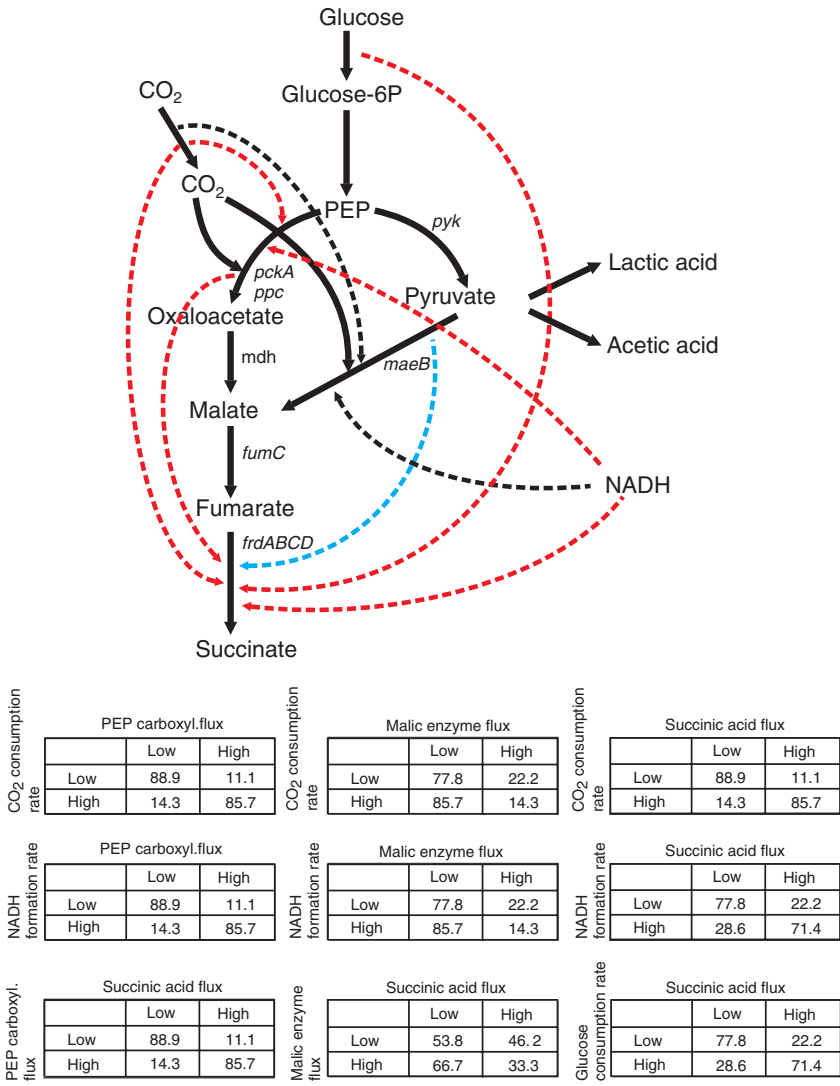

Figure 4 Effects of various metabolic fluxes on the succinic acid fluxes examined by Bayesian network analysis. Intracellular flux distributions obtained under $\mathrm{N}_{2}, \mathrm{CO}_{2}$ and $\mathrm{CO}_{2}-\mathrm{H}_{2}$ atmospheres at $\mathrm{pH} 5.5,6.0,6.5,7.0$ and 7.5 were used as input data, and the data were separated by two parts (high and low) according to the frequency. Red and blue dotted lines indicate positive and negative effects, respectively. The values in tables are conditional probabilities indicating the probability that $X$ is true while $Y$ is true, which can be defined as:

$$
P(X \mid Y)=P(X, Y) / P(Y)
$$

where $P(X, Y)$ is the joint probability inferred from the product of individual conditional probabilities $P(X)$ and $P(Y)$, which represent probabilities that $\mathrm{X}$ and $\mathrm{Y}$ are true, respectively. 
succinic acid pathway for $\mathrm{NAD}^{+}$regeneration in the rumen where $\mathrm{CO}_{2}$ is abundant.

The effect of $\mathrm{H}_{2}$ on the intracellular flux distribution was also evaluated by comparing the MFA results obtained under $\mathrm{CO}_{2}-\mathrm{H}_{2}$ (molar ratio of $1: 1$ ) versus $\mathrm{CO}_{2}$ (Fig. 3b). When $\mathrm{H}_{2}$ was present, cell growth rate and the glycolytic flux were lower. However, the relative succinic acid flux increased by $30 \%$ under $\mathrm{CO}_{2}-\mathrm{H}_{2}$, and the relative formation rates of ATP and $\mathrm{NADH}$ were higher in the presence of $\mathrm{H}_{2}$. The MFA results indicated that M. succiniciproducens consumes 2.8 moles of $\mathrm{CO}_{2}$ and 0.16 moles of $\mathrm{H}_{2}$ for the production of 4.0 moles of succinic acid. The succinic acid yield obtained under $\mathrm{CO}_{2}-\mathrm{H}_{2}$ was as high as 1.3 moles succinic acid/mole glucose, which is $70 \%$ of the theoretical succinic acid yield. Until now, it has been suggested that methanogens are responsible for the consumption of $\mathrm{H}_{2}$ to form methane from $\mathrm{CO}_{2}$ in the rumen ${ }^{14}$. However, the MFA results suggest that $M$. succiniciproducens, and possibly other capnophilic bacteria, may be important in controlling the rumen atmosphere through consumption of $\mathrm{CO}_{2}$ and $\mathrm{H}_{2}$ in conjunction with production of organic acids.

Examination of the overall metabolic flux profiles (Fig. 3c) obtained under various culture conditions suggests the following conclusions on the metabolic characteristics of M. succiniciproducens. The normalized glycolytic fluxes did not change much under varying culture conditions, suggesting the essential role of glycolytic fluxes on 을 the metabolic housekeeping activities of M. succiniciproducens by producing $50 \%-70 \%$ of total ATP and $77 \%-85 \%$ of NADH. The normalized fluxes of the TCA cycle and amino acid biosynthesis under $\mathrm{CO}_{2}$ were lower than those under $\mathrm{N}_{2}$. On the other hand, the normalized succinic acid flux considerably increased under $\mathrm{CO}_{2}$, which coincides with the increase of PEP carboxylation flux.

Bayesian network analysis showed that the $\mathrm{CO}_{2}$ consumption rate, PEP carboxylation flux and succinic acid flux showed a direct relationship with one another, suggesting that PEP carbolxylation is a major $\mathrm{CO}_{2}$-consuming, $\mathrm{C}_{3}-\mathrm{C}_{4}$ link in $M$. succiniciproducens leading to $\mathrm{NAD}^{+}$regeneration and succinic acid production (Fig. 4). Besides $\mathrm{CO}_{2}$, the glucose consumption rate and $\mathrm{NADH}$ formation rate also exerted a positive effect on succinic acid production. Malic enzyme flux did not affect succinic acid flux.

\section{DISCUSSION}

More than 145 microbial genome projects have been completed. They have mainly focused on the characterization of virulence-related genes and specific pathways or enzymes. Only recently, genome-scale in silico models are beginning to appear for E. coli ${ }^{15,16}$ and several other microorganisms ${ }^{17}$. In this study, we were able to decipher through the genome-scale MFA how the metabolism of M. succiniciproducens evolved and how it is regulated in response to variations in the conditions in the rumen. M. succiniciproducens possesses an efficient system for the carboxylation of PEP to oxaloacetate, which is further converted to succinic acid through the reductive TCA cycle and the menaquinone system. It was predicted by MFA that M. succiniciproducens can produce as much as 1.71 and 1.86 moles of succinic acid from 1 mole of glucose under $\mathrm{CO}_{2}$ and $\mathrm{CO}_{2}-\mathrm{H}_{2}$ atmospheres, respectively. Therefore, it will be possible to use this bacterium for the efficient production of succinic acid, an important industrial chemical that can be used as a green feedstock for the manufacture of biodegradable polymers, synthetic resins and various chemical intermediates and additives ${ }^{3}$. In conclusion, we were able to decipher the metabolic characteristics of M. succiniciproducens directly from the genome sequence by genome-scale MFA based on actual fermentation data. Based on these findings, we can now design metabolic engineering strategies for the enhanced production of succinic acid; one such strategy will be increasing the PEP carboxylation flux while decreasing the fluxes to acetic, formic and lactic acid. This approach will prove useful in developing metabolic engineering strategies for the production of various bioproducts by other sequenced organisms.

\section{METHODS}

Genome sequencing strategy. The M. succiniciproducens MBEL55E (KCTC 0769BP; the Korean Collection for Type Cultures) genome was shotgun sequenced $^{18}$. E. coli DH5 $\alpha$ (supE44, $\Delta$ lacU169 ( $\phi 80$ lacZ $\left.4 M 15\right)$, hsdR17, recA1, endA1, gyrA96, thi-1, relA1) was used for genomic DNA library construction. M. succiniciproducens was grown in Luria-Bertani medium ${ }^{19}$. Nine plasmid libraries of small inserts $(3-4 \mathrm{~kb})$ were constructed using pGEM$3 \mathrm{Zf}(+)$ (Applied Biosystems) and pUC18 (Amersham Biosciences) after random mechanical shearing of genomic DNA. A large insert library $(\sim 38.5 \mathrm{~kb})$ was constructed using the fosmid pEpiFOS-5 (Epicentre) after size selection of genomic DNA without additional shearing. Plasmid DNA was extracted from the cells grown in 96-well plates by the alkaline lysis method followed by purification using glass fiber-based filter plates. Sequencing was carried out using an ABI prism 3700 DNA analyzer (Applied Biosystems). A total of 36,820 reads with an average length of 614 bp ( $\sim 9.7$-fold coverage) were obtained by bidirectional sequencing. A total of 530 end reads from 275 fosmid clones served as genome scaffolds. The sequences were assembled using PHRAP (http://www.phrap.org/). Visual inspection of assembly results, primer design for gap closing and sequence editing were done using CONSED ${ }^{20}$. Possible misassemblies were corrected by validity checking on the directions and distances between the mate reads. Sequence gaps were closed by primer walking on plasmid or fosmid clones (1,875 primer walk reads), and physical gaps were closed by direct sequencing of genomic DNA or PCR products. The accuracy of the final consensus sequence was $99.987 \%$, including $1.8 \%$ edited bases.

ORF prediction and annotation. ORF prediction and annotation were done using the JAVA-based airBASE software package (KAIST-Bioinfomatix) installed on an IBM server, which integrated GLIMMER ${ }^{21}$, ARTEMIS ${ }^{22}$, BLAST $^{23}$ and Hidden Markov Models (HMM) modules ${ }^{24}$. Automatic analysis of each gene candidate was done using BLAST with COG data ${ }^{25}$ and HMM modules. The cut-off E-values of $10^{-5}$ and $10^{-1}$ were used in the BLAST and HMMpfam analyses, respectively. The tRNAs were predicted by tRNAscan-SE software $^{26}$. Genes assigned to multiple COGs were further analyzed to determine their primary functional categories.

Dot matrix plot analysis. The dot matrix plots comparing two genomes were generated by Genalysis software (http://www.genetix.com/productpages/ Software/Genalysis.htm). The output displays a 2-D plot representing matched regions between two genomes by colored dots. The minimum size of matched sequences was set to $20 \mathrm{bp}$.

Metabolic flux and computational analyses. MFA was carried out for the calculation of volumetric rates of intracellular metabolite formation using the in silico metabolic network constructed directly from the genome sequence. Because the number of reactions was greater than the number of metabolites, the system was underdetermined. Therefore, MFA was carried out by optimization using linear programming with an objective function of achieving the maximum growth rate ${ }^{27}$ :

Maximize (shown in wt \%): $0.495 \times$ protein $+0.25 \times \mathrm{RNA}+0.041 \times \mathrm{DNA}$ $+0.091 \times$ lipids $+0.034 \times$ lipopolysaccharides $+0.025 \times$ peptidoglycan + $0.025 \times$ glycogen $+0.004 \times$ polyamines $+0.035 \times$ cofactors.

In the above equation, the contents of protein, RNA and DNA in $M$. succiniciproducens were measured values, whereas those of other macromolecules were based on average E. coli cellular contents ${ }^{28}$. MFA was carried out using the program package MetaFluxNet ${ }^{29}$. The glucose uptake rate and the production rates of lactic, acetic, formic and succinic acids experimentally measured were used as the constraints during the MFA.

Even though flux analysis using linear programming is effective to describe the overall metabolic characteristics, there may be several flux scenarios when the objective function and/or applied constraints are altered. To examine if the metabolic characteristics deciphered by flux analysis above are reliable, we 
repeated flux analyses using several suboptimal growth rates as objective functions; we varied the objective values from $100 \%$ to $90 \%$ of the maximum objective value and monitored changes in flux distributions. Most of anabolic fluxes decreased with decreasing objective value, whereas the central metabolic fluxes in glycolysis and C3-C4 linkages were less affected by the changes in the objective value. Most importantly, the activation of alternative fluxes and reversal of flux direction were not observed during the suboptimal flux analysis, which suggests that the results obtained by flux analysis based on linear programming represent consistent and reliable information on the intracellular metabolism of M. succiniciproducens.

For the comparative analysis of respiratory systems in various microorganisms, hierarchical clustering was performed using the Cluster program package $^{30}$ with the average-linkage hierarchical clustering algorithm. The results were visualized using the TreeView software package ${ }^{30}$

Culture conditions. Cells were routinely grown in a $\mathrm{CO}_{2}$ atmosphere in sealed anaerobic bottles containing $100 \mathrm{ml}$ of $\mathrm{MH}$ medium $^{2}$ plus $10 \mathrm{~g} / \mathrm{l}$ of glucose. For flask cultures, exponentially growing cells, washed anaerobically with the $\mathrm{MH}$ medium, were used to inoculate sealed anaerobic bottles containing $100 \mathrm{ml}$ of $\mathrm{MH}$ medium plus $10 \mathrm{~g} / \mathrm{l}$ of glucose. Batch cultures were kept at $39^{\circ} \mathrm{C}$ in a jar fermentor (2.51; KoBioTech) containing 1 liter of $\mathrm{MH} 2$ medium plus $20 \mathrm{~g} / \mathrm{l}$ of glucose and $1 \mathrm{mg} / \mathrm{l}$ of $\mathrm{Na}_{2} \mathrm{~S} \cdot 9 \mathrm{H}_{2} \mathrm{O}$. MH2 medium contains per liter: $5 \mathrm{~g}$ yeast extract, $3 \mathrm{~g} \mathrm{~K}_{2} \mathrm{HPO}_{4}, 1 \mathrm{~g} \mathrm{NaCl}, 1 \mathrm{~g}\left(\mathrm{NH}_{4}\right)_{2} \mathrm{SO}_{4}, 0.2 \mathrm{~g} \mathrm{CaCl}_{2} \cdot 2 \mathrm{H}_{2} \mathrm{O}$ and $0.2 \mathrm{~g}$ $\mathrm{MgCl}_{2} \cdot 6 \mathrm{H}_{2} \mathrm{O}$. To examine the effect of $\mathrm{pH}$ on metabolism, the $\mathrm{pH}$ was controlled at a desired value between 5.5 and 7.5 using $5 \mathrm{~N} \mathrm{NaOH}$. Cells were cultured under three different atmospheric conditions: $\mathrm{CO}_{2}, \mathrm{CO}_{2}-\mathrm{H}_{2}$ mixture (molar ratio of 1:1) or $\mathrm{N}_{2}$ (ref. 2). Gases, scrubbed free of oxygen by a gas purifier (P.J. Cobert Associates), were sparged at 0.25 liter min $^{-1}$ during the cultivation. When bacteria were cultured under $\mathrm{CO}_{2}$ and $\mathrm{CO}_{2}-\mathrm{H}_{2}$ atmospheres, $7 \mathrm{~g}$ of $\mathrm{Na}_{2} \mathrm{CO}_{3}$ was added to prevent culture acidification. The agitation speed 을 was 200 r.p.m.

Analytical procedure. The concentrations of glucose and fermentation products were determined by HPLC (Hitachi L-3300 RI detector, D2500 chromato-integrator) equipped with an ion exchange column (Aminex HPX-87H, $300 \mathrm{~mm} \times 7.8 \mathrm{~mm}$ ) using $0.012 \mathrm{~N} \mathrm{H}_{2} \mathrm{SO}_{4}$ as a mobile phase ${ }^{2}$. Cell growth was monitored by measuring the absorbance at $660 \mathrm{~nm}\left(\mathrm{OD}_{660}\right.$; Ultrospec 3000 , Pharmacia Biotech). The protein concentration was measured by the Bradford method $^{31}$. The DNA and RNA contents were determined using the Burton and orcinol methods, respectively ${ }^{32}$

Nucleotide sequence accession number. The sequence reported in this paper To has been deposited in GenBank under the accession number AE016827.

Note: Supplementary information is available on the Nature Biotechnology website.

\section{ACKNOWLEDGMENTS}

We thank Sung Ho Goh and Heyung Ju Shin at the KRIBB, Young Ho Moon, Kang Ryul Choi, Sun Ho Cha, Sung Soo Kim, Soo Hyun Jeong, Eun Mi Chung and Sun Rye Jung at GenoTech, and Jinyoung Park, Sujin Chae and Hee Sun Chung at Bioinfomatix for their contributions during genome sequencing and annotation. This work was supported by the Korean Systems Biology Research Program (M10309020000-03B5002-00000) of the Ministry of Science and Technology (MOST), Bioinfomatix and by the Brain Korea 21 Project. Further support through the LG Chem Chair Professorship and IBM SUR program is appreciated.

\section{COMPETING INTERESTS STATEMENT}

The authors declare that they have no competing financial interests.

Received 7 June; accepted 3 August 2004

Published online at http://www.nature.com/naturebiotechnology/

1. Sniffen, C.J. \& Herdt, H.H. The Veterinary Clinics of North America: Food Animal Practice (W.B. Saunders Company, Pennsylvania, USA, 1991).
2. Lee, P.C., Lee, S.Y., Hong, S.H. \& Chang, H.N. Isolation and characterization of a new succinic acid-producing bacterium, Mannheimia succiniciproducens MBEL55E, from bovine rumen. Appl. Microbiol. Biotechnol. 58, 663-668 (2002).

3. Lee, S.Y., Hong, S.H., Lee, S.H. \& Park, S.J. Fermentative production of chemicals that can be used for polymer synthesis. Macromol. Biosci. 4, 157-164 (2004).

4. Salzberg, S.L., Salzberg, A.J., Kerlavage, A.R. \& Tomb, J.F. Skewed oligomers and origins of replication. Gene 217, 57-67 (1998).

5. Blattner, F.R. et al. The complete genome sequence of Escherichia coli K-12. Science 277, 1453-1462 (1997).

6. Kunst, F. et al. The complete genome sequence of the gram-positive bacterium Bacillus subtilis. Nature 390, 249-256 (1997).

7. May, B.J. et al. Complete genomic sequence of Pasteurella multocida, Pm70. Proc. Natl. Acad. Sci. USA 98, 3460-3465 (2001).

8. Kanehisa, M., Goto, S., Kawashima, S. \& Nakaya, A. The KEGG databases at GenomeNet. Nucleic Acids Res. 30, 42-46 (2002).

9. Karp, P.D. Pathway databases: a case study in computational symbolic theories. Science 293, 2040-2044 (2001)

10. Fleischmann, R.D. et al. Whole-genome random sequencing and assembly of Haemophilus influenzae Rd. Science 269, 496-512 (1995).

11. Angen, Ø., Mutters, R., Caugant, D.A., Olsen, J.E. \& Bisgaard, M. Taxonomic relationships of the [Pasteurella] haemolytica complex as evaluated by DNA-DNA hybridizations and 16S rRNA sequencing with proposal of Mannheimia haemolytica gen. nov. comb. nov., Mannheimia granulomatis comb. nov., Mannheimia glucosida sp. nov. Mannheimia ruminalis sp. nov. and Mannheimia varigena sp. nov. Int. J. Syst. Bacteriol. 49, 67-86 (1999).

12. Osawa, R. et al. Lonepinella koalarum gen. nov., sp. nov., a new tannin-protein complex degrading bacterium. Syst. Appl. Microbiol. 18, 368-373 (1995).

13. Guettler, M.V., Rumler, D. \& Jain, M.K. Actinobacillus succinogenes sp. nov., a nove succinic-acid-producing strain from the bovine rumen. Int. J. Syst. Bacteriol. 49, 207 216 (1999)

14. Church, D.C. The Ruminant Animal: Digestive Physiology and Nutrition (Prentice Hall, New Jersey, USA, 1988).

15. Ibarra, R.U., Edwards, J.S. \& Palsson, B.O. Escherichia coli K-12 undergoes adaptive evolution to achieve in silco predicted optimal growth. Nature 420, 186-189 (2002).

16. Edwards, J.S., Ibarra, R.U. \& Palsso, B.O. In silico predictions of Escherichia coli metabolic capabilities are consistent with experimental data. Nat. Biotechnol. 19, 125-130 (2001)

17. Price, N.D., Papin, J.A., Schilling, C.H. \& Palsson, B.O. Genome-scale microbial in silico models: the constraints-based approach. Trends Biotechnol. 21, 162-169 (2003).

18. Favello, A., Hillier, L. \& Wilson, R.K. Genomic DNA sequencing methods. Methods Cell Biol. 48, 551-569 (1995).

19. Sambrook, J., Fritsch, E.F. \& Maniatis, T. Molecular Cloning (Cold Spring Harbor Laboratory Press, Cold Spring Harbor, New York, 1989).

20. Gordon, D., Abajian, C. \& Green, P. Consed: a graphical tool for sequence finishing. Genome Res. 8, 195-202 (1998)

21. Delcher, A.L., Harmon, D., Kasif, S., White, O. \& Salzberg, S.L. Improved microbial gene identification with GLIMMER. Nucleic Acids Res. 27, 4636-4641 (1999).

22. Rutherford, K. et al. Artemis: sequence visualization and annotation. Bioinformatics 16, 944-945 (2000).

23. Altschul, S.F., Gish, W., Miller, W., Myers, E.W. \& Lipman, D.J. Basic local alignment search tool. J. Mol. Biol. 215, 403-410 (1990).

24. Eddy, S.R. Profile hidden Markov models. Bioinformatics 14, 755-763 (1998).

25. Tatusov, R.L. et al. The COG database: new developments in phylogenetic classification of proteins from complete genomes. Nucleic Acids Res. 29, 22-28 (2001).

26. Lowe, T.M. \& Eddy, S.R. tRNAscan-SE: a program for improved detection of transfer RNA genes in genomic sequence. Nucleic Acids Res. 25, 955-964 (1997).

27. Varma, A. \& Palsson, B.O. Metabolic flux balancing-basic concepts, scientific and practical use. Bio/Technology 12, 994-998 (1994).

28. Neidhardt, F.C. \& Umbarger, H.E. in Escherichia coli and Salmonella: Cellular and Molecular Biology, edn. 2 (ed. Neidhardt, F.C.) 13-16 (ASM Press, Washington, DC 1996)

29. Lee, D.Y., Yun, H., Park, S. \& Lee, S.Y. MetaFluxNet: the management of metabolic reaction information and quantitative metabolic flux analysis. Bioinformatics 19 , 2144-2146 (2003).

30. Eisen, M.B., Spellman, P.T., Brown, P.O. \& Botstein, D. Cluster analysis and display of genome-wide expression patterns. Proc. Natl. Acad. Sci. USA 95, 14863-14868 (1998).

31. Bradford, M.M. A rapid and sensitive method for the quantitation of microgram quantities of protein utilizing the principle of protein-dye binding. Anal. Biochem. 72, 248-254 (1976)

32. Burton, K. A study of the conditions and mechanism of the diphenylamine reaction for the colorimetric estimation of deoxyribonucleic acid. Biochem. J. 62, 314-323 (1956). 